\title{
Portfolio Diversification under Local and Moderate Deviations from Power Laws.
}

\section{Citation}

Ibragimov, Rustam, and Johan Walden. 2008. Portfolio diversification under local and moderate deviations from power laws. Insurance: Mathematics and Economics 42(2): 594-599.

\section{Published Version}

http://dx.doi.org/10.1016/j.insmatheco.2007.06.006

\section{Permanent link}

http://nrs.harvard.edu/urn-3:HUL.InstRepos:2640586

\section{Terms of Use}

This article was downloaded from Harvard University's DASH repository, and is made available under the terms and conditions applicable to Other Posted Material, as set forth at http:// nrs.harvard.edu/urn-3:HUL.InstRepos:dash.current.terms-of-use\#LAA

\section{Share Your Story}

The Harvard community has made this article openly available.

Please share how this access benefits you. Submit a story.

Accessibility 


\title{
PORTFOLIO DIVERSIFICATION UNDER LOCAL AND MODERATE DEVIATIONS FROM POWER LAWS ${ }^{1}$
}

\author{
Rustam Ibragimov \\ Department of Economics, Harvard University \\ Johan Walden \\ Haas School of Business, University of California at Berkeley
}

Address for manuscript correspondence:

\author{
Rustam Ibragimov \\ Department of Economics \\ Harvard University \\ Littauer Center \\ 1875 Cambridge St. \\ Cambridge, MA 02138 \\ Email: ribragim@fas.harvard.edu \\ Phone: +1-617-496-4795 \\ Fax: +1-617-495-7730 \\ Johan Walden \\ Haas School of Business \\ University of California at Berkeley \\ 545 Student Services Building, \#1900 \\ Berkeley, CA 94720-1900 \\ Email: walden@haas.berkeley.edu \\ Phone: +1-510-643-0547 \\ Fax: +1-510-643-1420
}

\footnotetext{
${ }^{1}$ An extended version of this work is available as Ibragimov \& Walden (2006). We are grateful to Donald Andrews, Xavier Gabaix, Peter Phillips, Herbert Scarf, Michael Schwarz and the participants at seminars at the Departments of Economics at Yale University, University of British Columbia, the University of California at San Diego, Harvard University, the London School of Economics and Political Science, Massachusetts Institute of Technology, the Université de Montréal, McGill University and New York University, the Division of the Humanities and Social Sciences at California Institute of Technology, Nuffield College, University of Oxford, and the Department of Statistics at Columbia University for helpful discussions. We also thank an anonymous referee for many useful comments and suggestions. The support of the NUS Risk Management Institute is gratefully acknowledged.
} 


\begin{abstract}
This paper analyzes portfolio diversification for nonlinear transformations of heavy-tailed risks. It is shown that diversification of a portfolio of convex functions of heavy-tailed risks increases the portfolio's riskiness, if expectations of these risks are infinite. On the contrary, for concave functions of heavy-tailed risks with finite expectations, the stylized fact that diversification is preferable continues to hold. The framework of transformations of heavy-tailed risks includes many models with Pareto-type distributions that exhibit local or moderate deviations from power tails in the form of additional slowly varying or exponential factors. The class of distributions under study is therefore extended beyond the stable class.
\end{abstract}

KEYWORDS: heavy-tailed risks; nonlinear transformations; portfolios; diversification; riskiness; value at risk; risk bounds; Pareto-type distributions; power laws; local and moderate deviations; domain of attraction of stable distributions

\title{
JEL Classification: G11
}

\section{Introduction}

In the recent four decades, we have witnessed a rapid expansion of the study of heavy-tailedness and the extreme outliers phenomena in economics and finance. Beginning with Mandelbrot (1963) and Fama $(1965 b)$, numerous studies have documented that time series encountered in many fields in economics and finance are typically heavy-tailed and have infinite moments of order $p \geq \alpha$ for certain $\alpha>0$ (see the discussion in Loretan \& Phillips 1994, Gabaix, Gopikrishnan, Plerou \& Stanley 2003, Ibragimov 2004a,b, 2005, Rachev, Menn \& Fabozzi 2005, Ibragimov \& Walden 2007, and references therein).

In models involving a heavy-tailed cdf $F$ with infinite moments of order greater than or equal to $\alpha$, it is typically assumed that $F$ has Pareto (power) tails:

$$
F(x)=\frac{c_{1}+o(1)}{|x|^{\alpha}}, x \rightarrow-\infty ; 1-F(x)=\frac{c_{2}+o(1)}{x^{\alpha}}, x \rightarrow+\infty,
$$

or, more generally, that $F$ is of Pareto-type, so that

$$
F(x)=\frac{c_{1}+o(1)}{|x|^{\alpha}} l(|x|), \quad x \rightarrow-\infty ; 1-F(x)=\frac{c_{2}+o(1)}{x^{\alpha}} l(x), \quad x \rightarrow+\infty .
$$

Here, $c_{1}, c_{2}$ are some positive constants and $l(x)$ is a slowly varying function at infinity: $l(\lambda x) / l(x) \rightarrow 1$, as $x \rightarrow+\infty$, for all $\lambda>0$, and $\alpha$ is the so-called tail index. Well-known examples of distributions satisfying (1) are stable laws with $\alpha \in(0,2)$, that is, distributions that are closed under portfolio formation (see Section 2).

We mention a sample of estimates of the tail index $\alpha$ for returns on various stocks and stock indices: $3<\alpha<5$ (Jansen \& de Vries 1991), $2<\alpha<4$ (Loretan \& Phillips 1994), $1.5<\alpha<2$ (McCulloch 1997), $0.9<\alpha<2$ (Rachev \& Mittnik 2000), $\alpha \approx 3$ (Gabaix et al. 2003). As discussed by 
Nešlehova, Embrechts \& Chavez-Demoulin (2006), tail indices less than one are observed for empirical loss distributions of a number of operational risks. Furthermore, Scherer, Harhoff \& Kukies (2000) and Silverberg \& Verspagen (2004) report the tail indices $\alpha$ to be considerably less than one for financial returns from technological innovations. Rachev et al. (2005) discuss and review the vast literature that supports heavy-tailedness and Pareto distributions for equity and bond returns. ${ }^{2}$

As was shown in Ibragimov $(2004 a, b, 2005)$ in a general context based on majorization theory and arbitrary portfolio weights comparisons, diversification may be inferior for convolutions of stable extremely heavy-tailed and possibly dependent risks whose cdf's $F$ satisfy power law (1) with $\alpha<1$. According to Ibragimov $(2004 a, b, 2005)$, diversification is typically preferable for convolutions of stable heavy-tailed risks that follow (1) with $\alpha>1$. Recently, Ibragimov \& Walden (2007) showed that, with bounded risks concentrated on a sufficiently large interval, diversification may be suboptimal up to a certain number of risks and then become optimal. The above findings generalize the results in Fama (1965a), Samuelson (1967) and Ross (1976) on portfolio choice in the stable framework and riskiness analysis for portfolios of stable risks with equal weights. Several examples that illustrate the phenomenon that diversification is not always preferable are presented in Kaas, Goovarets \& Tang (2004).

Clearly, understanding the conditions under which diversification is preferable is core to fields such as finance, banking and insurance. Stable distributions form a small subclass of the class of Paretotype laws and the previous examples of heavy-tailed distributions may not belong to this subclass. A natural question is therefore whether the stylized facts about riskiness and portfolio diversification can be extended beyond the subclass of stable distributions. The main objective of this paper is to carry out such an extension. We analyze portfolio diversification for nonlinear transformations of heavy-tailed risks, to understand under what distributional assumptions failure of diversification occurs. We use the framework of value at risk as a measure of portfolio riskiness.

Our contribution to the literature is two-fold. First, we show that diversification of a portfolio of convex functions of heavy-tailed risks increases its riskiness if expectations of these risks are infinite (Theorem 2). However, the stylized fact that diversification is always preferable continues to hold for concave (on $\mathbf{R}_{+}$) functions of heavy-tailed risks with finite expectations (Theorem 1).

Second, we use the results on nonlinear transformations to model a large class of Pareto-type distributions. The class of nonlinear transformations of heavy-tailed r.v.'s considered in this paper provides a natural framework for modeling risks with distributions exhibiting departures from power laws. Specifically, let us define recursively the iterations of a logarithm by $\ln _{0}(x)=x, \ln _{k}(x)=\ln \left[\ln _{k-1}(x)\right], k \geq 1$, for all large positive $x$. Let $m \geq 0$ and let $\gamma_{1}, \ldots, \gamma_{m} \in \mathbf{R}$ be some constants. The stochastic framework considered in the paper covers risks $Y$ whose tails behave as

$$
P(|Y|>x) \asymp x^{-\alpha} \prod_{i=1}^{m}\left[\ln _{i}(x)\right]^{\gamma_{i}}=x^{-\alpha}(\ln x)^{\gamma_{1}}(\ln \ln x)^{\gamma_{2}} \ldots(\ln \ln \ldots \ln x)^{\gamma_{m}}, x \rightarrow \infty
$$

\footnotetext{
${ }^{2}$ Recently, using the framework of nonlinear transformations of r.v.'s, Malevergne \& Sornette (2003) have focused on the analysis of value at risk $(\mathrm{VaR})$ and other measures of risk for the class of heavy-tailed risks with Weibull-like (stretched exponential) distribution. Contrary to our analysis, these tails decay faster than any power law (1) (see also Malevergne \& Sornette 2004b,a, for a review of properties of Weibull-type distributions).
} 
(here and throughout the paper, $g(x) \asymp h(x)$ as $x \rightarrow \infty$ denotes that there are constants, $c$ and $C$ such that $0<c \leq g(x) / h(x) \leq C<\infty$ for large $x>0)$. In particular, the choice $\gamma_{k}=-1, \gamma_{s}=0$, $1 \leq s \leq m, s \neq k$, produces deviations from power law (1) of the form $P(|Y|>x) \asymp \frac{1}{x^{\alpha} \ln _{k}(x)}$. Similarly, the choice $\gamma_{k}=1, \gamma_{s}=0,1 \leq s \leq m, s \neq k$ corresponds to the deviations from power law (1) of the form $P(|Y|>x) \asymp \frac{\ln _{k}(x)}{x^{\alpha}}$. Also, when $\alpha=1$ the above relations correspond to deviations from the Zipf law (1) of the form $P(|Y|>x) \asymp \frac{1}{x \ln _{k}(x)} ; P(|Y|>x) \asymp \frac{\ln _{k}(x)}{x}$. Reminiscent of the terminology in the time series unit root literature (see Phillips 1988, Phillips \& Magdalinos 2004), it is natural to refer to distributions whose tails satisfy one of relation (3) as exhibiting "local" or "moderate" deviations from power laws. Corollaries 1 and 2 provide results for when diversification will and will not be preferred for such deviations. We also show that the results above can not be generalized to the whole class of Pareto-type distributions (see Examples 2 and 1 in In Subsection 3.1). In order to highlight the main ideas and concepts discussed, the results in this paper are formulated in the framework of independent risks, which is the case where diversification works best. One can obtain analogous results for wide classes of dependent risks, including those with $\alpha$-symmetric distributions and convolutions of models with common shocks. The extension to dependent risks is quite straightforward, and is similar to the extensions in Ibragimov (2004a,b, 2005), Ibragimov \& Walden (2007).

The paper is organized as follows. Section 2 introduces classes of distributions we are dealing with throughout the paper and discusses their main properties. Section 3.1 presents our main results on diversification and its effects on the value at risk of portfolios of nonlinear transformations of heavytailed risks. Section 3.2 discusses implications of the results for local and moderate deviations from power laws in form (3). Finally, Section 4 makes some concluding remarks. All proofs are left to the Appendix.

\section{Notations and classes of distributions}

We say that a r.v. $X$ with density $p: \mathbf{R} \rightarrow \mathbf{R}$ and the convex distribution support $\Omega=\{x \in \mathbf{R}: p(x)>$ $0\}$ is log-concavely distributed if $\log p(x)$ is concave in $x \in \Omega$, that is, if for all $x_{1}, x_{2} \in \Omega$, and any $\lambda \in[0,1], p\left(\lambda x_{1}+(1-\lambda) x_{2}\right) \geq\left(p\left(x_{1}\right)\right)^{\lambda}\left(p\left(x_{2}\right)\right)^{1-\lambda}$ (see An 1998). A distribution is said to be log-concave if its density $p$ satisfies the above inequalities. Examples of log-concave distributions include the normal, uniform, exponential and logistic distributions, the Gamma distribution $\Gamma(\alpha, \beta)$ with $\alpha \geq 1$, the Beta distribution $\mathcal{B}(a, b)$ with $a \geq 1$ and $b \geq 1$, and the Weibull distribution $\mathcal{W}(\gamma, \alpha)$ with $\alpha \geq 1$. If a r.v. $X$ is log-concavely distributed, then its density has at most an exponential tail, that is, $p(x)=o(\exp (-\lambda x))$ for some $\lambda>0$, as $x \rightarrow \infty$ and all the power moments $E|X|^{\gamma}, \gamma>0$, of the r.v. exist (see Corollary 1 in An 1998). We denote by $\mathcal{L C}$ the class of symmetric log-concave distributions.

For $0<\alpha \leq 2, \sigma>0, \beta \in[-1,1]$ and $\mu \in \mathbf{R}$, we denote by $S_{\alpha}(\sigma, \beta, \mu)$ the stable distribution with the index of stability $\alpha$, the scale parameter $\sigma$, the skewness parameter $\beta$ and the location parameter 
$\mu$. That is, $S_{\alpha}(\sigma, \beta, \mu)$ is the distribution of a r.v. $X$ with the characteristic function

$$
E\left(e^{i x X}\right)= \begin{cases}\exp \left\{i \mu x-\sigma^{\alpha}|x|^{\alpha}(1-i \beta \operatorname{sign}(x) \tan (\pi \alpha / 2))\right\}, & \alpha \neq 1, \\ \exp \{i \mu x-\sigma|x|(1+(2 / \pi) i \beta \operatorname{sign}(x) \ln |x|)\}, & \alpha=1,\end{cases}
$$

$x \in \mathbf{R}$, where $i^{2}=-1$ and $\operatorname{sign}(x)$ is the sign of $x$ defined by $\operatorname{sign}(x)=1$ if $x>0, \operatorname{sign}(0)=0$ and $\operatorname{sign}(x)=-1$ otherwise. In what follows, we write $X \sim S_{\alpha}(\sigma, \beta, \mu)$, if the r.v. $X$ has the stable distribution $S_{\alpha}(\sigma, \beta, \mu)$. A closed form expression for the density $f(x)$ of the distribution $S_{\alpha}(\sigma, \beta, \mu)$ is available only in the following cases: $\alpha=2$ (Gaussian distributions); $\alpha=1$ and $\beta=0$ (Cauchy distributions); $\alpha=1 / 2$ and $\beta= \pm 1$ (Lévy distributions). If $X \sim S_{\alpha}(\sigma, \beta, \mu)$, then its distribution satisfies power law (1). Distributions $S_{\alpha}(\sigma, \beta, \mu)$ with $\mu=0$ for $\alpha \neq 1$ and $\beta=0$ for $\alpha=1$ are called strictly stable. If $X_{i} \sim S_{\alpha}(\sigma, \beta, \mu), \alpha \in(0,2]$, are i.i.d. strictly stable r.v.'s, then, for all $a_{i} \geq 0, i=1, \ldots, n$, with $\sum_{i=1}^{n} a_{i} \neq 0$, we have $\sum_{i=1}^{n} a_{i} X_{i} /\left(\sum_{i=1}^{n} a_{i}^{\alpha}\right)^{1 / \alpha} \sim S_{\alpha}(\sigma, \beta, \mu)$ (see Zolotarev (1986), Embrechts, Klüppelberg \& Mikosch (1997) and Rachev \& Mittnik (2000) for a detailed review of properties of stable distributions).

Let $\mathbf{R}_{+}=[0, \infty)$. Throughout the paper, $\bar{M}$ denotes the class of differentiable odd functions $f: \mathbf{R} \rightarrow$ $\mathbf{R}$ such that $f$ is concave and increasing on $\mathbf{R}_{+}$and $\underline{M}$ denotes the class of odd functions $f: \mathbf{R} \rightarrow \mathbf{R}$ such that $f$ is convex and increasing on $\mathbf{R}_{+}$. Further, $\bar{M}^{\prime}$ (resp. $\underline{M}^{\prime}$ ) denotes the subclass of $\bar{M}$ (resp. $\underline{M}$ ) consisting of functions $f$ which are strictly concave (strictly convex) on $\mathbf{R}_{+}$.

By $\overline{\mathcal{C T} \mathcal{S L C}}$, we denote the class of convolutions of log-concave distributions and distributions of transforms $f(Y), f \in \bar{M}$, of symmetric stable r.v.'s $Y \sim S_{\alpha}(\sigma, 0,0)$ with characteristic exponents $\alpha \in[1,2]$ and $\sigma>0$. In what follows, we write $X \sim \mathcal{L C}$ (resp., $X \sim \overline{\mathcal{C} \mathcal{T S L C}}$ ) if the distribution of the r.v. $X$ belongs to the class $\mathcal{L C}$ (resp., $\overline{\mathcal{C} \mathcal{T} \mathcal{S L C}}$ ). The class $\overline{\mathcal{C} \mathcal{T} \mathcal{S L C}}$ thus consists of distributions of r.v.'s $X$ such that, for some $k \geq 1$, and independent r.v.'s $Y_{0} \sim \mathcal{L C}$ and $Y_{i} \sim S_{\alpha_{i}}\left(\sigma_{i}, 0,0\right), \alpha_{i} \in[1,2], \sigma_{i}>0$, $i=1, \ldots, k$,

$$
X=\theta Y_{0}+f_{1}\left(Y_{1}\right)+\ldots+f_{k}\left(Y_{k}\right)
$$

where $\theta \in\{0,1\}, f_{i} \in \bar{M}$, if $\alpha_{i}>1$, and $f_{i} \in \bar{M}^{\prime}$ if $\alpha_{i}=1, i=1, \ldots, k$. (It will follow from our analysis that $\alpha_{i}=1$ is a special case, for which the assumption $f_{i} \in \bar{M}^{\prime}$ is needed for the value at risk comparisons to be strict).

\section{Main results}

\subsection{Diversification of nonlinear transformations of heavy-tailed risks}

Let $0<q<1 / 2$. Given a r.v. (risk) $Z$, we denote by $\operatorname{VaR}_{q}[Z]$ the value at risk (VaR) of $Z$ at level $q$, that is, its $(1-q)$-quantile: $\operatorname{VaR}_{q}[Z]=\inf \{z \in \mathbf{R}: P(Z>z) \leq q\}$ (throughout the paper, we interpret the positive values of $Z$ as a risk holder's losses). For $n \geq 1$, the sample mean $\bar{X}_{n}$ represents the return on 
the portfolio of risks $X_{1}, \ldots, X_{n}$ with equal weights $w_{n}=(1 / n, 1 / n, \ldots, 1 / n): \bar{X}_{n}=\frac{1}{n} \sum_{i=1}^{n} X_{i}$. Theorem 1 shows that diversification continues to be preferable for convex transformations of heavy-tailed risks with finite expectations.

Theorem 1 The following conclusions hold.

- Let $Y_{1} \sim S_{\alpha}(\sigma, \beta, 0), \alpha \in[1,2], \sigma>0, \beta \in[-1,1]$. Further, let $X_{1}$ and $X_{2}$ be i.i.d risks such that $X_{1}=f\left(Y_{1}\right)$, where $f \in \bar{M} ; f \in \bar{M}^{\prime}$ for $\alpha=1$. Then $\operatorname{VaR}_{q}\left[\bar{X}_{2}\right]<\operatorname{VaR}_{q}\left[X_{1}\right]$.

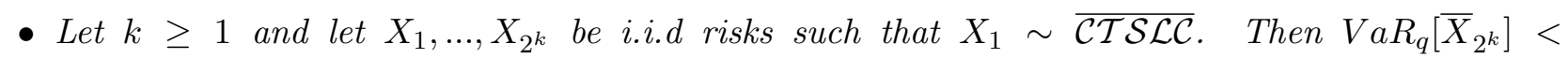
$\operatorname{VaR}_{q}\left[\bar{X}_{2^{k-1}}\right]<\ldots<\operatorname{VaR} R_{q}\left[X_{1}\right]$.

On the contrary, Theorem 2 demonstrates that the stylized fact on diversification being preferable is reversed for concave (on $\mathbf{R}_{+}$) transformations of heavy-tailed risks with infinite expectations.

Theorem 2 Let $Y_{1} \sim S_{\alpha}(\sigma, \beta, 0), \alpha \in(0,1], \sigma>0, \beta \in[-1,1]$. Further, let $X_{1}$ and $X_{2}$ be i.i.d. risks such that $X_{1}=f\left(Y_{1}\right)$, where $f \in \underline{M} ; f \in \underline{M^{\prime}}$ for $\alpha=1$. Then $\operatorname{VaR} R_{q}\left[X_{1}\right]<\operatorname{VaR} q\left[\bar{X}_{2}\right]$.

These results may seem intuitive, as $\alpha=1$ is the value at which the preferability of diversification is reversed for stable distributions (see Ibragimov 2004a,b, 2005, Ibragimov \& Walden 2007). For example, in the case of i.i.d. symmetric Cauchy risks $X_{i} \sim S_{1}(\sigma, 0,0), i=1, \ldots, n$, we have $\bar{X}_{n} \sim S_{1}(\sigma, 0,0)$, so that diversification has no effect on the portfolio VaR. However, outside the class of stable distributions the effects of diversification on portfolio riskiness are not that simple. In fact, the intuition that distributions with thinner tails than Cauchy-type power laws (equations (1) with $\alpha=1$ ) are always "good" to diversify and distributions with heavier tails are always "bad," may not hold. Let us give two examples:

Example 1 Risks with heavier tails than those of Cauchy distributions, where diversification may be preferable: An example of dependent risks with such properties is provided by the following construction. Consider risks $U_{i}$ given by $U_{i}=Z X_{i}, i=1, \ldots, 2^{k}$, where $X_{i}=f\left(Y_{i}\right), f \in \bar{M}^{\prime}, Y_{i} \sim S_{1}(\sigma, 0,0)$ are i.i.d. symmetric Cauchy r.v.'s and $Z>0$ is a positive r.v. independent of $Y_{i}^{\prime}$ s. This is an example of a model with a common shock $Z$ that affects all dependent risks $U_{i}$. The risks $U_{i}$ have tails that are heavier than those of Cauchy distributions with $\alpha=1$ in the case of common shocks $Z$ with infinite first moments: $E Z=\infty$. However, using Theorem 1 and conditioning arguments, we get that $V a R_{q}\left[\bar{U}_{2^{k}}\right]<$ $\operatorname{VaR} R_{q}\left[\bar{U}_{2^{k-1}}\right]<\ldots<\operatorname{Va} R_{q}\left[U_{1}\right]$, so diversification is preferable.

Example 2 Risks with thinner tails than those of Cauchy distributions, where diversification may not be preferable: Consider i.i.d. risks $X_{1}, X_{2}$ such that $X_{i}=f\left(Y_{i}\right)$, where $f \in \underline{M}^{\prime}$ and $Y_{i} \sim S_{1}(\sigma, 0,0)$ are i.i.d. symmetric Cauchy r.v.'s. In particular, as discussed in the introduction and in the next subsection, the above setup models risks with tails exhibiting deviations from power laws (1) in the form 
$P\left(\left|X_{i}\right|>x\right) \asymp \frac{\ln _{k}(x)}{x}$. Further, let $Z_{i}, i=1,2$, be i.i.d. symmetric stable risks with the tail index $\alpha>1$, say $\alpha=1.1: Z_{i} \sim S_{\alpha}(\sigma, 0,0)$, so that the tails of their distributions decline to zero faster than those of Cauchy distributions. Given $A>0$, define the risks $Y_{i}^{(A)}=X_{i} I\left(\left|X_{i}\right|<A\right)+Z_{i} I\left(\left|Z_{i}\right|>A\right)$, where $I(\cdot)$ stands for the indicator function. Clearly, the tails of distributions of the risks $Y_{i}^{(A)}$ behave like those of $Z_{i}: P\left(\left|Y_{i}^{(A)}\right|>x\right) \asymp P\left(\left|Z_{i}\right|>x\right) \asymp \frac{1}{x^{\alpha}}$ for large $x$ and are thus thinner than the tails of Cauchy distributions with $\alpha=1$.

From Theorem 2 it follows that $\operatorname{VaR}_{q}\left[X_{1}\right]<\operatorname{VaR}_{q}\left[\frac{1}{2}\left(X_{1}+X_{2}\right)\right]$. Since, evidently, $Y_{i}^{(A)} \rightarrow X_{i}$ (in distribution) as $A \rightarrow \infty$, this implies that there exists a sufficiently large $A$ such that diversification can not be said to be optimal for the risks $Y_{i}^{(A)}$ with thinner tails than those of Cauchy distributions: $\operatorname{VaR}_{q}\left[Y_{1}^{(A)}\right]<\operatorname{VaR} R_{q}\left[\frac{1}{2}\left(Y_{1}^{(A)}+Y_{2}^{(A)}\right)\right]$.

\subsection{Diversification and value at risk under deviations from power laws}

As indicated in the introduction, the class of nonlinear transformations of heavy-tailed r.v.'s considered in this paper provides a natural framework for modeling risks with distributions exhibiting local to moderate departures from power laws in forms (2) and (3).

Let $m \geq 0, \gamma_{1}, \ldots, \gamma_{m} \in \mathbf{R}$. Further, let $x_{0}$ be a large positive constant. Consider the odd increasing on $\mathbf{R}$ function $V$ defined by

$$
V(x)=\left(x+x_{0}\right) \prod_{i=1}^{m}\left[\ln _{i}\left(x+x_{0}\right)\right]^{\gamma_{i} / \alpha}-x_{0} \prod_{i=1}^{m}\left[\ln _{i}\left(x_{0}\right)\right]^{\gamma_{i} / \alpha}, \quad V(-x)=-V(x), \quad x>0 .
$$

For $k \in\{1,2, \ldots, m\}$ and sufficiently large $x$ we have that the function $G(x)=x \prod_{i=k}^{m} g_{i}(x)$, where $g_{i}(x)=\left(\ln _{i}(x)\right)^{\gamma_{i} / \alpha}$, satisfies

$$
\frac{G^{\prime \prime}(x)}{g_{k}^{\prime}(x) \prod_{i=k+1}^{m} g_{i}(x)}=\sum_{i=k}^{m} \frac{g_{i}^{\prime}(x) g_{k}(x)}{g_{k}^{\prime}(x) g_{i}(x)}+\sum_{i=k}^{m}\left(1+\frac{x g_{i}^{\prime \prime}(x)}{g_{i}^{\prime}(x)}\right) \frac{g_{i}^{\prime}(x) g_{k}(x)}{g_{k}^{\prime}(x) g_{i}(x)}+\sum_{k \leq i<j \leq m} \frac{g_{i}^{\prime}(x) g_{k}(x)}{g_{k}^{\prime}(x) g_{i}(x)} \cdot \frac{x g_{j}^{\prime}(x)}{g_{j}(x)} .
$$

Let us show, using (5), that

$$
\frac{G^{\prime \prime}(x)}{g_{k}^{\prime}(x) \prod_{i=k+1}^{m} g_{i}(x)}=1+o(1)
$$

as $x \rightarrow \infty$. Since

$$
g_{i}^{\prime}(x)=\frac{\left(\gamma_{i} / \alpha\right) g_{i}(x)}{x \prod_{j=1}^{i} \ln _{j}(x)}
$$

we get that $g_{i}^{\prime}(x)=o\left(\frac{g_{i}(x)}{x}\right)$ as $x \rightarrow \infty$ for $k \leq i \leq m$, and $\frac{g_{i}^{\prime}(x)}{g_{i}(x)}=o\left(\frac{g_{k}^{\prime}(x)}{g_{k}(x)}\right)$ as $x \rightarrow \infty$ for $k+1 \leq i \leq m$. To show that $1+\frac{x g_{i}^{\prime \prime}(x)}{g_{i}^{\prime}(x)}=o(1)$ as $x \rightarrow \infty$ for all $k \leq i \leq m$, we rewrite $1+\frac{x g_{i}^{\prime \prime}(x)}{g_{i}^{\prime}(x)}=1+x \frac{d\left(\ln \left[g_{i}^{\prime}(x)\right]\right)}{d x}$ which by (7) is equal to $1+x \frac{d}{d x}\left(\ln \left(\gamma_{i} / \alpha\right)+\ln \left[g_{i}(x)\right]-\ln (x)-\sum_{j=2}^{i} \ln _{j}(x)\right)=x \frac{g_{i}^{\prime}(x)}{g_{i}(x)}-\sum_{j=2}^{i} x \frac{d}{d x}\left[\ln _{j}(x)\right]$. The 
first term in this expression is $o(1)$ as shown above, and, as $\frac{d}{d x}\left[\ln _{j}(x)\right]=\left(x \prod_{k=1}^{j-1} \ln _{k}(x)\right)^{-1}$ for $j \geq 2$, each of the other summands is also $o(1)$. From (5) and the above relations it follows that (6) indeed holds and, consequently, $G^{\prime \prime}(x)$ (and, therefore, $V^{\prime \prime}(x)$ ) has the same sign as $g_{k}^{\prime}(x)$ for sufficiently large $x$.

This implies that the function $V(x)$ is strictly convex on $[0, \infty)$ for a sufficiently large $x_{0}$ if $\gamma_{1}=0, \ldots$, $\gamma_{k-1}=0, \gamma_{k}>0$ for some $k \in\{1,2, \ldots, m\}$. Similarly, $V(x)$ is strictly concave on $[0, \infty)$ for a sufficiently large $x_{0}$ if $\gamma_{1}=0, \ldots, \gamma_{k-1}=0, \gamma_{k}<0$ for some $k \in\{1,2, \ldots, m\}$. In addition, since, for $Y=V(Z)$, $P(|Y|>x)=P\left(|Z|>V^{-1}(x)\right)$ and $V^{-1}(x) \asymp x \prod_{i=1}^{m}\left[\ln _{i}(x)\right]^{-\gamma_{i} / \alpha}$ as $x \rightarrow \infty$, one gets that the distribution of $Y$ satisfies (3) if $Z$ is a r.v. whose cdf $F$ follows power law (1).

The results on nonlinear transformations of heavy-tailed risks imply Corollaries 1 and 2 below. These corollaries concern the analysis of portfolio diversification under local, and moderate deviations from power laws in form (3). For instance, Corollary 1 demonstrates that the stylized facts on portfolio diversification continue to hold for portfolios of nonlinear transformations of risks with the tail index $\alpha \geq 1$ whose distributions have tails that satisfy (3) with $\alpha>1$ or with $\alpha=1, \gamma_{1}=0, \ldots, \gamma_{k-1}=0$, $\gamma_{k}>0$ for some $k \in\{1,2, \ldots, m\}$. Thus, Corollary 1 implies that portfolio diversification decreases portfolio riskiness in the world of risks whose distributions are even slightly thinner than those of Cauchy r.v.'s.

Corollary 1 Let the parameters of the function $V$ be such that $\gamma_{1}=0, \ldots, \gamma_{k-1}=0, \gamma_{k}<0$ for some $k \in\{1,2, \ldots, m\}$. Further, let $x_{0}$ in the definition of $V$ be sufficiently large so that the function is welldefined, odd and increasing on $\mathbf{R}$ and is strictly concave on $[0, \infty)$. If $X_{1}$ and $X_{2}$ are i.i.d risks such that $X_{1}=V\left(Y_{1}\right)$, where $Y_{1} \sim S_{\alpha}(\sigma, \beta, 0), \alpha \in[1,2], \sigma>0, \beta \in[-1,1]$, then the conclusions of Theorem 1 hold.

However, Corollary 2 implies that diversification always leads to an increase in riskiness for portfolios of nonlinear functions of risks with $\alpha \leq 1$ whose distributions have tails satisfying (3) with $\alpha<1$ or with $\alpha=1, \gamma_{1}=0, \ldots, \gamma_{k-1}=0, \gamma_{k}<0$ for some $k \in\{1,2, \ldots, m\}$. Thus, Corollary 2 shows that riskiness of a portfolio increases with diversification for risks with distributions whose tails are even slightly heavier than those of Cauchy r.v.'s.

Corollary 2 Let the parameters of the function $V$ be such that $\gamma_{1}=0, \ldots, \gamma_{k-1}=0, \gamma_{k}>0$ for some $k \in\{1,2, \ldots, m\}$. Further, let $x_{0}$ in the definition of $V$ be sufficiently large so that the function is welldefined, odd and increasing on $\mathbf{R}$ and is strictly convex on $[0, \infty)$. If $X_{1}$ and $X_{2}$ are i.i.d risks such that $X_{1}=V\left(Y_{1}\right)$, where $Y_{1} \sim S_{\alpha}(\sigma, \beta, 0), \alpha \in(0,1], \sigma>0, \beta \in[-1,1]$, then the conclusions of Theorem 2 hold. 


\section{Concluding remarks}

The concept of diversification is a corner stone of financial theory. Understanding when diversification is preferable is therefore important. This paper provides an extended analysis of this issue beyond the class of stable distributions. It is an empirical question whether the Pareto-type behavior of economic and financial data, discussed in the introduction, falls within this extended class. This question is outside the scope of the current paper, but should be addressed by future research.

\section{Appendix - Proofs}

Proof of Theorems 1 and 2. Let $\alpha_{1} \in[1,2], \alpha_{2} \in(0,1]$, and let $f_{1} \in \bar{M}, f_{2} \in \underline{M}$. Suppose that $f_{1} \in \bar{M}^{\prime}$ if $\alpha_{1}=1$ and $f_{2} \in \underline{M}^{\prime}$ if $\alpha_{2}=1$. For $j=1,2$, let $Y_{1}^{(j)}$ and $Y_{2}^{(j)}$ be i.i.d. r.v.'s such that $Y_{i}^{(j)} \sim S_{\alpha_{j}}(\sigma, \beta, 0)$, $\sigma>0, \beta \in[-1,1], i=1,2$, and let $X_{i}^{(j)}=f_{j}\left(Y_{i}^{(j)}\right), i=1,2, j=1,2$. As in the proof of Lemmas 2.7 and 2.8 in Proschan (1965), by the definition of the classes $\bar{M}$ and $\underline{M}$ we have that

$$
\begin{gathered}
\left|f_{1}\left(\left(y_{1}+y_{2}\right) / 2\right)\right| \geq\left|\left(f_{1}\left(y_{1}\right)+f_{1}\left(y_{2}\right)\right) / 2\right|, \\
\left|f_{2}\left(\left(y_{1}+y_{2}\right) / 2\right)\right| \leq\left|\left(f_{2}\left(y_{1}\right)+f_{2}\left(y_{2}\right)\right) / 2\right|
\end{gathered}
$$

for all $y_{1}, y_{2} \in \mathbf{R}$. In addition, inequality (8) is strict for $y_{1}+y_{2} \neq 0$ if $f_{1} \in \bar{M}^{\prime}$. Similarly, inequality (9) is strict for $y_{1}+y_{2} \neq 0$ if $f_{2} \in \underline{M}^{\prime}$. Since the functions $\left|f_{j}(x)\right|, j=1,2$, are increasing in $|x|$, we, therefore, get that

$$
\begin{aligned}
& \left|f_{1}\left(\left(y_{1}+y_{2}\right) / 2^{1 / \alpha_{1}}\right)\right| \geq\left|\left(f_{1}\left(y_{1}\right)+f_{1}\left(y_{2}\right)\right) / 2\right|, \\
& \left|f_{2}\left(\left(y_{1}+y_{2}\right) / 2^{1 / \alpha_{2}}\right)\right| \leq\left|\left(f_{2}\left(y_{1}\right)+f_{2}\left(y_{2}\right)\right) / 2\right| .
\end{aligned}
$$

Obviously, inequality in (10) is strict for $y_{1}+y_{2} \neq 0$ if $\alpha_{1}>1$ and inequality in (11) is strict for $y_{1}+y_{2} \neq 0$ if $\alpha_{2}<1$. Since $2^{-1 / \alpha_{j}}\left(Y_{1}^{(1)}+Y_{2}^{(1)}\right) \sim S_{\alpha_{j}}(\sigma, \beta, 0), j=1,2$, and the functions $f_{j}, j=1,2$, are odd, this implies that, for all $q \in(0,1 / 2)$,

$$
\begin{array}{r}
\operatorname{VaR}_{q}\left[\frac{X_{1}^{(1)}+X_{2}^{(1)}}{2}\right]=\operatorname{Va} R_{q}\left[\frac{f_{1}\left(Y_{1}^{(1)}\right)+f_{1}\left(Y_{2}^{(1)}\right)}{2}\right]< \\
\operatorname{VaR}_{q}\left[f_{1}\left(\frac{Y_{1}^{(1)}+Y_{2}^{(1)}}{2^{1 / \alpha_{1}}}\right)\right]=\operatorname{Va} R_{q}\left[f_{1}\left(Y_{1}^{(1)}\right)\right]=\operatorname{Va} R_{q}\left[X_{1}^{(1)}\right]
\end{array}
$$

and

$$
\begin{array}{r}
\operatorname{VaR}_{q}\left[\frac{X_{1}^{(2)}+X_{2}^{(2)}}{2}\right]=\operatorname{Va} R_{q}\left[\frac{f_{2}\left(Y_{1}^{(2)}\right)+f_{2}\left(Y_{2}^{(2)}\right)}{2}\right]> \\
\operatorname{VaR}_{q}\left[f_{2}\left(\frac{Y_{1}^{(2)}+Y_{2}^{(2)}}{2^{1 / \alpha_{2}}}\right)\right]=\operatorname{VaR} R_{q}\left[f_{2}\left(Y_{1}^{(2)}\right)\right]=\operatorname{VaR}\left[R_{q}\left[X_{1}^{(2)}\right] .\right.
\end{array}
$$


According to relation (12), the part of Theorem 1 for transformations of stable r.v.'s holds. Relation (13) shows that Theorem 2 is true.

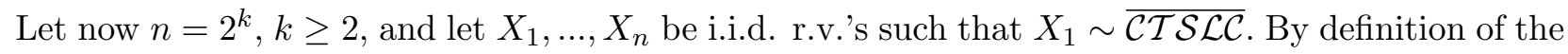
class $\overline{\mathcal{C T} \mathcal{S} \mathcal{L C}}$, there exist i.i.d. r.v.'s $Y_{i t}, t=0,1, \ldots, k, i=1, \ldots, n$, and functions $f_{t} \in \bar{M}, t=1, \ldots, k$, such that $Y_{i 0} \sim \mathcal{L C}$ and $Y_{i t} \sim S_{\alpha_{t}}\left(\sigma_{t}, 0,0\right), \alpha_{t} \in(1,2], \sigma_{t}>0, t=1, \ldots, k$, and $X_{i}=\theta Y_{i 0}+f_{1}\left(Y_{i 1}\right)+\ldots+f_{k}\left(Y_{i k}\right)$, $\theta \in\{0,1\}, i=1, \ldots, n$. From (12) we have that for all $i=1, \ldots, n / 2$ and $t=1, \ldots, k$,

$$
\operatorname{VaR}_{q}\left[\frac{f_{t}\left(Y_{i t}\right)+f_{t}\left(Y_{n / 2+i, t}\right)}{2}\right]<\operatorname{VaR}_{q}\left[f_{t}\left(Y_{i t}\right)\right] .
$$

In addition, by Proschan (1965),

$$
\operatorname{VaR}_{q}\left[\frac{Y_{i 0}+Y_{n / 2+i, 0}}{2}\right]<\operatorname{VaR} q\left[f_{t}\left(Y_{i 0}\right)\right] .
$$

According to Theorem 2.7.6 in Zolotarev (1986), p. 134, and Theorem 1.10 in Dharmadhikari \& Joag-Dev (1988), p. 20, the densities of the r.v.'s $Y_{i t}, t=0,1, \ldots, k, i=1, \ldots, n$, are symmetric and unimodal. This implies, as it is not difficult to see, symmetry and unimodality of the densities of the r.v.'s $f_{t}\left(Y_{i t}\right), f_{t} \in \bar{M}, t=1, \ldots, k, i=1, \ldots, n$. By Theorem 1.6 in Dharmadhikari \& Joag-Dev (1988), p. 13, we get, in turn, that the densities of the r.v.'s $\left(Y_{i 0}+Y_{n / 2+i, 0}\right) / 2$ and $\left(f_{t}\left(Y_{i t}\right)+f_{t}\left(Y_{n / 2+i, t}\right)\right) / 2$, $t=1, \ldots, k, i=1, \ldots, n$, are symmetric and unimodal.

From Lemma in Birnbaum (1948) and its proof it follows that if $\xi_{1}, \xi_{2}$ and $\eta_{1}, \eta_{2}$ are independent absolutely continuous symmetric unimodal r.v.'s such that, for $j=1,2$, and all $q \in(0,1 / 2), V_{a} R_{q}\left[\xi_{j}\right]<$ $\operatorname{VaR}_{q}\left[\eta_{j}\right]$, then $\operatorname{Va} R_{q}\left[\xi_{1}+\xi_{2}\right]<\operatorname{VaR}_{q}\left[\eta_{1}+\eta_{2}\right], q \in(0,1 / 2)$. This, together with (14) and (15), implies by induction (see also Theorem 1 in Birnbaum, 1948, and Theorem 2.C.3 in Dharmadhikari and Joag-Dev, 1988) that

$$
\begin{gathered}
\operatorname{VaR}_{q}\left[\bar{X}_{n}\right]=\operatorname{VaR}_{q}\left[\frac{1}{n} \sum_{i=1}^{n / 2}\left\{\theta\left(Y_{i 0}+Y_{n / 2+i, 0}\right)+\left(f_{1}\left(Y_{i 1}\right)+f_{1}\left(Y_{n / 2+i, 1}\right)\right) \ldots+\left(f_{k}\left(Y_{i k}\right)+f_{k}\left(Y_{n / 2+i, k}\right)\right)\right\}\right]< \\
\operatorname{VaR} q\left[\frac{2}{n} \sum_{i=1}^{n / 2}\left\{\theta Y_{i 0}+f_{1}\left(Y_{i 1}\right)+\ldots+f_{k}\left(Y_{i k}\right)\right\}\right]=\operatorname{VaR} R_{q}\left[\bar{X}_{n / 2}\right] .
\end{gathered}
$$

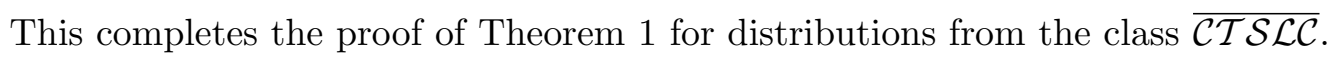

Proof of Corollaries 1 and 2. Corollary 1 follows from Theorem 1 since, under the assumptions of the corollary, the function $V$ belongs to the class $f \in \bar{M}^{\prime}$. Similarly, Corollary 2 is a consequence of Theorem 2 and the fact that, under its assumptions, the function $V$ belongs to the class $f \in \underline{M}^{\prime}$.

\section{References}

An, M. Y. (1998), 'Logconcavity versus logconvexity: a complete characterization', Journal of Economic

Theory 80, 350-369. 
Birnbaum, Z. W. (1948), 'On random variables with comparable peakedness', Annals of Mathematical Statistics 19, 76-81.

Dharmadhikari, S. W. \& Joag-Dev, K. (1988), Unimodality, convexity and applications, Boston: Academic Press.

Embrechts, P., Klüppelberg, C. \& Mikosch, T. (1997), Modelling extremal events for insurance and finance, Springer, New York.

Fama, E. (1965a), 'Portfolio analysis in a stable Paretian market', Management Science 11, 404-419.

Fama, E. F. (1965b), 'The behavior of stock market prices', Journal of Business 38, 34-105.

Gabaix, X., Gopikrishnan, P., Plerou, V. \& Stanley, H. E. (2003), 'A theory of power-law distributions in financial market fluctuations', Nature 423, 267-270.

Ibragimov, R. (2004a), 'On the robustness of economic models to heavy-tailedness assumptions', Mimeo, Yale University . Available at http://post.economics.harvard.edu/faculty/ibragimov/ Papers/HeavyTails.pdf.

Ibragimov, R. (2004b), 'Portfolio diversification and value at risk under thick-tailedness', Harvard University Research Discussion Paper 2086 . Available at http://post.economics.harvard.edu/hier/ 2005papers/HIER2086.pdf.

Ibragimov, R. (2005), New majorization theory in economics and martingale convergence results in econometrics, Ph.D. dissertation, Yale University.

Ibragimov, R. \& Walden, J. (2006), 'Portfolio diversification under local, moderate and global deviations from power laws', Harvard University Research Discussion Paper 2116. Available at http://www.economics.harvard.edu/hier/2006papers/HIER2116.pdf.

Ibragimov, R. \& Walden, J. (2007), 'The limits of diversification when losses may be large', Journal of Banking and Finance. In Press. http://dx.doi.org/10.1016/j.jbankfin.2006.11.014. Also available as Harvard Institute of Economic Research Discussion Paper No. 2104. http://www.economics.harvard.edu/hier/2006papers/HIER2104.pdf.

Jansen, D. W. \& de Vries, C. G. (1991), 'On the frequency of large stock returns: Putting booms and busts into perspective', Review of Economics and Statistics 73, 18-32.

Kaas, R., Goovarets, M. \& Tang, Q. (2004), 'Some useful counterexamples regarding comonotonicity', Belgian Actuarial Bulletin 4, 1-4.

Loretan, M. \& Phillips, P. C. B. (1994), 'Testing the covariance stationarity of heavy-tailed time series', Journal of Empirical Finance 1, 211-248.

Malevergne, Y. \& Sornette, D. (2003), 'VaR-efficient portfolios for a class of super- and sub-exponentially decaying assets return distributions', Quantitative Finance 4, 17-36. 
Malevergne, Y. \& Sornette, D. (2004a), 'High-order moments and cumulants of multivariate Weibull asset returns distributions: analytical theory and empirical tests: II', Finance Letters 3, 54-63.

Malevergne, Y. \& Sornette, D. (2004b), 'Multivariate Weibull Distributions for Asset Returns: I', Finance Letters 2, 16-32.

Mandelbrot, B. (1963), 'The variation of certain speculative prices', Journal of Business 36, 394-419.

McCulloch, J. H. (1997), 'Measuring tail thickness to estimate the stable index alpha: A critique', Journal of Business and Economic Statistics 15, 74-81.

Nešlehova, J., Embrechts, P. \& Chavez-Demoulin, V. (2006), 'Infinite mean models and the LDA for operational risk', Journal of Operational Risk 1, 3-25.

Phillips, P. C. B. (1988), 'Regression theory for near-integrated time series', Econometrica 56, 10211043.

Phillips, P. C. B. \& Magdalinos, T. (2004), 'Limit theory for moderate deviations from a unit root', Cowles Foundation Discussion Paper 1471 . Available at http://cowles.econ.yale.edu/P/cd/d14b/ d1471.pdf.

Proschan, F. (1965), 'Peakedness of distributions of convex combinations', Annals of Mathematical Statistics 36, 1703-1706.

Rachev, S. T., Menn, C. \& Fabozzi, F. J. (2005), Fat-tailed and skewed asset return distributions: implications for risk management, portfolio selection, and option pricing, Wiley, Hoboken, NJ.

Rachev, S. T. \& Mittnik, S. (2000), Stable Paretian Models in Finance, Wiley, New York.

Ross, S. A. (1976), 'A note on a paradox in portfolio theory', Working paper, University of Pennsylvania

Samuelson, P. A. (1967), 'Efficient portfolio selection for Pareto-Lévy investments', Journal of Financial and Quantitative Analaysis 2, 107-122.

Scherer, F. M., Harhoff, D. \& Kukies, J. (2000), 'Uncertainty and the size distribution of rewards from innovation', Journal of Evolutionary Economics 10, 175-200.

Silverberg, G. \& Verspagen, B. (2004), 'The size distribution of innovations revisited: an application of extreme value statistics to citation and value measures of patent significance', Working paper, Maastricht University 36, 1703-1706.

Zolotarev, V. M. (1986), One-dimensional stable distributions, Providence: American Mathematical Society. 